HNO 2019 $\cdot 67: 326-333$

https://doi.org/10.1007/s00106-019-0664-0

Online publiziert: 18. April 2019

(c) Der/die Autor(en) 2019

\author{
A. Meppelink \\ Brainlab AG, München, Deutschland
}

\title{
Digitaler OP: bessere Planung und Kommunikation
}

tralen Op.-Cockpitsystem zur Steuerung von Bild-, Planungs- und Videoquellen sind dies moderne Navigationsverfahren, automatisierte, effiziente Softwareanwendungen zur Op.-Planung sowie roboterbasierte Assistenzsysteme. Wesentliche Effekte dieser Neuentwicklungen sind die präzisere Ausführung des chirurgischen Plans, effizientere Prozesse vor, während und nach der Op. und somit mehr Zeit und Sicherheit für den Patienten.

Das Potenzial dieser Innovationen im Klinikalltag zu nutzen, stellt angesichts der Komplexität eines OP eine große Herausforderung dar - auch deshalb, weil mit fortschreitender technischer Entwicklung die Anzahl spezieller Geräte im $\mathrm{OP}$ zunimmt. Die heutigen Op.Säle sind häufig überfüllt mit freistehenden Geräten, unterstützenden Systemen und Monitoren, die alle über eine eigene Schnittstelle oder Hardware zur Datenanzeige verfügen. Dies macht es schwierig, einen klaren Überblick über alle Daten zu behalten. Zudem lenkt diese Vielfalt die Aufmerksamkeit des Op.-Teams weg vom Patienten.

Im OP der Zukunft geht es jedoch nicht nur um die Einbindung medizinischer Hightech-Geräte, sondern auch um Datenanreicherung und Informationsintegration. Op.-Teams sehen sich heute der Herausforderung gegenüber, immer mehr Technologie und Informationen sinnvoll und klinisch relevant zu kombinieren und dabei die Op.-Zeiten inklusive Vor- und Nachbereitung nicht zu verlängern. Außerdem verändern sich die technologischen Möglichkeiten rasant und die Verantwortlichen müssen sicherstellen, dass Innovationen mit echtem Mehrwert für die Patienten skalierbar sind und möglichst einfach und schnell in bestehende Systeme integriert werden können.

Einer der Vorreiter bei Entwicklung, Konzeption und Installation digitaler OP ist die Fa. Brainlab, München. Das Münchner Unternehmen entwickelt seit 30 Jahren softwarebasierte Medizintechnik für die Chirurgie und bietet auch Komplettlösungen für digital integrierte Op.-Säle. Diese multidisziplinären Lösungen sind in weit mehr als 1000 Op.Sälen weltweit im Einsatz.

\section{》) Der digitale OP nutzt das Potenzial diagnostischer, chirurgischer und postoperativer Patientendaten}

Eine gute Voraussetzung für einen umfassend digitalisierten OP ist eine offene, modulare, softwarebasierte Plattform. Eine solche Infrastruktur berücksichtigt neben den Anforderungen der Abteilungen für Informationstechnologie (IT) auch die Forderung der Chirurgen und Op.-Teams nach einfacher Bedienbarkeit, individualisierbaren, schnellen Setups, flexiblen Darstellungs- und Aufbereitungsmöglichkeiten der klinisch relevanten Daten sowie umfassenden Kommunikations- und Dokumentationsmöglichkeiten.

Diese Anforderungen sind im einfachen allgemeinchirurgischen $\mathrm{OP}$ ebenso zu erfüllen wie in einem komplexen Hybrid-OP - und nicht nur dort: Eine komplette Digitalisierung hilft auch dabei, vor dem Eingriff Patienten aufzuklären oder die Op. vorab mit Kollegen zu besprechen. Intuitive Benutzeroberflächen, automatisierte Arbeitsabläufe und erweiterte Bildgebungsverfahren zeichnen einen 
Hier steht eine Anzeige.

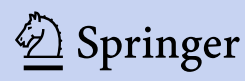


digitalen OP aus. Dank dieser Technologien können Chirurgen das Potenzial diagnostischer, chirurgischer und postoperativer Patientendaten voll ausschöpfen.

Doch das Konzept eines digitalisierten OP beinhaltet heute technische Standards, die weit mehr verlangen als lediglich die Konsolidierung von Op.-Informationen in einer einzigen Datenquelle oder einer zentralen Schnittstelle für das Bilddatenarchivierungs- und Kommunikationssystem („picture archiving and communication system", PACS), das Krankenhaus-Informationssystem (KIS) und andere im Krankenhaus vorhandene Archivierungssysteme. $\mathrm{Zu}$ einer umfassenden Integration gehören innovative Software-Anwendungen, die den Chirurgen bei der Optimierung von Patientenbildern sowie bei der sekundenschnellen Erstellung und Umsetzung des bestmöglichen chirurgischen Plans unterstützen.

\section{Patientensicherheit}

Ein Blick auf den Alltag eines chirurgisch tätigen HNO-Arztes verdeutlicht das Potenzial, das ein digitalisierter OP auch bei sehr häufig vorgenommenen Eingriffen birgt. Ein Beispiel dafür sind die deutschlandweit jährlich mehr als 40.000 Nasennebenhöhlenoperationen [5]: Trotz der damit einhergehenden Routine bergen die oft kurzen Operationen die Gefahr schwerster Komplikationen, wie zum Beispiel die Verletzung des Sehnervs oder des Gehirns. Um Qualität und Sicherheit auf einem konstant hohen Niveau zu halten, steht HNO-Operateuren mittlerweile ein umfassendes Portfolio an Planungs- und Navigationssystemen zur Verfügung. Dazu gehören erweiterte 3-D-Displays, berührungslose Patientenregistrierungsoptionen sowie die automatisierte Erkennung anatomischer Strukturen in Echtzeit.

\section{I) Chirurgische Planung sorgt auch bei Routineeingriffen für mehr Patientensicherheit}

Chirurgische Planung sorgt auch bei Routineeingriffen für mehr Patientensicherheit: Die chirurgische Planungs- technologie unterstützt den Chirurgen dabei, komplexe anatomische Strukturen leichter zu evaluieren und Krankheitsbilder genauer und schneller bestimmen. Die patientenspezifische 3-D-Planung kann auch noch unmittelbar vor der Op. vorgenommen werden. Sie dient dem Chirurgen während des Eingriffs als Unterstützung direkt im OP [2]. Die softwaregestützte chirurgische Planung im OP wird deutlich erleichtert, wenn eine Technologie verfügbar ist, die unabhängig von der Radiologie arbeitet, PACS-Patientenbilder lädt, von fremder Software erstellte Patientensegmentierung importiert, in 3-D-Format aufbereitet und Informationen fusioniert [3].

Im OP ist der zentrale Zugriff auf diese Daten v. a. kurz vor dem Eingriff notwendig, um den Plan nochmals zu validieren und ggf. finale Änderungen wie beispielsweise Messungen vorzunehmen. Dies gilt ebenso bei Notoperationen oder Eingriffen, die schnelles Handeln erfordern. Diese können mit der benutzerfreundlichen Software und durch schnelles Laden von Patientenbildern aus dem PACS zügig geplant werden. Die Praxis zeigt, dass Planungsdaten dann effizient und regelmäßig genutzt werden, wenn der für den nächsten Tag benötigte Op.Plan über Nacht automatisch vom KIS sowie die Patientenbilder vom PACS geladen und so Wartezeiten vor der Op. vermieden werden.

\section{I) Anhand von CT- und MRT- Aufnahmen lassen sich "on the fly" im OP 3-D-Bilder generieren}

Anhand von CT- und MRT-Aufnahmen lassen sich spontan („on the fly“) im OP 3-D-Bilder generieren: Für eine präzisere Planung des Eingriffs stehen dem Operateur heute innovative Software-Lösungen zur Verfügung, die anhand von CTund MRT-Aufnahmen ein dreidimensionales Bild des zu operierenden Bereichs generieren können. So ist es dem Chirurgen beispielsweise vom Büro aus möglich, mit der Planungssoftware Tumoren und Risikostrukturen in 3-D-Patientenbildern genau einzuzeichnen, Trajektorien zu planen sowie CT- und MRT-Bilder $\mathrm{zu}$ fusionieren. Die vorgeplanten Daten des chirurgischen Eingriffs werden dann automatisch an ein PACS oder KIS gesendet und können über eine HL7- und DICOM-Integration oder über eine CloudLösung wie beispielsweise Quentry sofort auf dem System im OP verwendet werden.

Eine weitere hilfreiche Funktion ist eine automatisierte Atlassegmentierung. Sie unterstützt Chirurgen dabei, klinische Fälle, zum Beispiel in der HNO, aber auch in komplexen neurochirurgischen Fällen, gleich zu Beginn umfassend zu bewerten. Auf Basis eines anpassungsfähigen anatomischen Software-Modells lassen sich je nach Modalität eine Vielzahl anatomischer Strukturen erkennen und als Objekte erstellen (• Abb. 1). Die hohe Genauigkeit und Konsistenz der Objekte machen die Segmentierung zu einem elementaren Bestandteil präziser Behandlungsplanung.

Mithilfe eines interaktiven digitalen Pinsels lässt sich etwa $\mathrm{zu}$ entfernendes Gewebe oder kritische Strukturen wie beispielsweise Sehnerv, Schädelbasis oder Karotiden präzise markieren. Vor einer Op. kann der Arzt am Computer per Maus oder auf einem Touchscreen die sichtbaren Konturen des betreffenden Gewebes entlangfahren. Hierbei funktioniert der digitale Stift so wie bei einem Grafikprogramm. Die Software erkennt automatisch und präzise die Konturen der gesuchten Strukturen und markiert die exakten Grenzen der Kontur auf Basis der groben Pinselführung des Chirurgen. Das Besondere ist, dass der Chirurg den digitalen Pinsel nur in 2 verschiedenen Ebenen führen muss. Das so markierte Objekt wird von der Software automatisch bis $\mathrm{zu}$ seinen Grenzen präzise ergänzt, ohne dass ein Einzeichnen in jeder einzelnen Schicht erforderlich ist. Zusätzlich wird ein dreidimensionales, farblich abgesetztes Modell des definierten Bereichs errechnet. Diese Bilder lassen sich dann im OP auf den verschiedenen Monitoren oder sogar im Okular des Mikroskops anzeigen und erscheinen damit ebenso im Sichtfeld des Operateurs wie die von ihm bewegte Spitze des Instruments.

Innovative Technologien der HNONavigation beinhalten eine präzise, 
schrittweise Anleitung zur berührungslosen Patientenregistrierung, die unabhängig von dessen Positionierung erfolgt. Hierzu wird ein Laser eingesetzt, der die Gesichtsoberfläche abtastet. Mithilfe der gewonnenen Punkte stellt ein SoftwareAlgorithmus den Bezug zwischen den diagnostischen Daten und der Anatomie des Patienten her und erstellt ein Oberflächenprofil, das wiederum dem Patientendatensatz zugeordnet wird. Da die Registrierung von CT- und MRTBildern ohne Rahmen oder Marker möglich ist, lassen sich zusätzliche Registrierscans vermeiden. Dies reduziert sowohl die Kosten für die Navigation als auch die Strahlenbelastung für den Patienten und das Op.-Team.

\section{Optische oder elektro- magnetische Navigation?}

Im Vorfeld einer Op. lässt sich mit moderner Software die präoperative Planung schnell und präzise an die intraoperative Navigation anpassen. Chirurgen können so den gesamten Eingriff virtuell vorplanen und unter bildgestützter Anleitung ausführen. Darüber hinaus kann die Navigation während des Eingriffs für die Ergebnisverifizierung verwendet werden.

\section{) HNO-Navigationssoftware ermöglicht in veränderten anatomischen Strukturen eine genaue Orientierung}

Vor allem bei Revisionseingriffen ermöglicht eine moderne HNO-Navigationssoftware auch in bereits veränderten anatomischen Strukturen, in denen Landmarken nur schwer oder überhaupt nicht erkennbar sind, eine genaue Orientierung. Besonders relevant ist die Op.Navigation bei Tumoroperationen, insbesondere in der Schädelbasischirurgie, wenn HNO-Ärzte und Neurochirurgen gemeinsam transsphenoidal operieren. Nicht selten arbeiten beide Fächer mit unterschiedlichen Instrumentarien wie speziellen Op.-Mikro- und -Endoskopen. Bei multidisziplinären Eingriffen etwa dieser beiden Fächer sind solche Navigationssysteme vorteilhaft, mit

HNO 2019 · 67:326-333 https://doi.org/10.1007/s00106-019-0664-0

(c) Der/die Autor(en) 2019

\section{A. Meppelink}

\section{Digitaler OP: bessere Planung und Kommunikation}

\section{Zusammenfassung}

Im Mittelpunkt der digitalen HNO-Chirurgie stehen v. a. 4 technologische Fortschritte: Neben dem Potenzial eines digitalisierten Operationssaals (OP) sind dies moderne Navigationsverfahren, Planungssoftware sowie roboterbasierte Assistenzsysteme. Im OP der Zukunft spielen nicht nur Datenanreicherung und Informationsintegration eine Rolle, sondern auch die Integration medizinischer Hightech-Geräte. Deren Nutzung und die von ihnen gelieferten Informationen werden vereinfacht; gleichzeitig jedoch werden weitere klinische Daten vor, während und nach dem chirurgischen Eingriff optimiert. Mit speziell entwickelten intuitiven Benutzeroberflächen, automatisierten Arbeitsabläufen und erweiterten Bildgebungsverfahren lässt sich das Potenzial diagnostischer, chirurgischer und postoperativer Patientendaten voll ausschöpfen. Chirurgische Planungstechnologie hilft HNO-Operateuren, komplexe anatomische Strukturen leichter zu evaluieren und Krankheitsbilder genauer und schneller zu bestimmen. Auf Basis eines anpassungsfähigen anatomischen Software-Modells lässt sich eine Vielzahl anatomischer Strukturen erkennen und als Objekte erstellen. Die hohe Genauigkeit und Konsistenz der Objekte machen die Segmentierung zu einem elementaren Bestandteil präziser Behandlungsplanung. Mit Planungssoftware lassen sich Tumoren und Risikostrukturen in 3-D-Patientenbildern genau einzeichnen, Trajektorien planen sowie CT- und MRT-Bilder fusionieren und anschließend im OP darauf für die weitere Nutzung einfach zurückgreifen. Dies dient während des Eingriffs als Anleitung und letztlich höherer Patientensicherheit sowie effizienteren Prozessen im klinischen Alltag.

\section{Schlüsselwörter}

Computerassistierte Chirurgie - Roboterassistierte Chirurgie - Medizinische Bildgebung . Integrierte digitale Operationssäle · OPDokumentation

\section{Digital OR: better planning and communication}

\section{Abstract}

At the center of digital ENT surgery are four primary technological developments: in addition to the potential of a digitalized operating room (OR), these are modern navigation approaches, planning software, and robotics-based assistance systems. In the OR of the future, not only will data enrichment and information integration play a role, but so will the incorporation of high-tech medical devices. Their use and the information they generate are simplified, while at the same time, further clinical data are optimized before, during, and after the surgical procedure. Specially designed intuitive user interfaces, automated workflows, and advanced imaging techniques allow the full potential of diagnostic, surgical, and postoperative patient data to be exploited. New surgical planning technology helps ENT surgeons to more easily evaluate complex anatomical structures and more accurately and quickly diagnose conditions. Based on an adaptable anatomical software model, a multitude of anatomical structures can be identified and segmented as objects. The high accuracy and consistency of identification of these objects make segmentation an integral part of precise treatment planning. With modern planning software, in 3D patient images, tumors and structures at risk can be accurately contoured, trajectories planned, and CT and MR images fused and subsequently easily accessed in the OR for further use. This serves as a guide during surgery and ultimately results in improved patient safety and more efficient routine clinical workflows.

\section{Keywords}

Computer assisted surgery - Robotic surgery . Medical Imaging · Integrated digital Operating Room - OR Documentation 


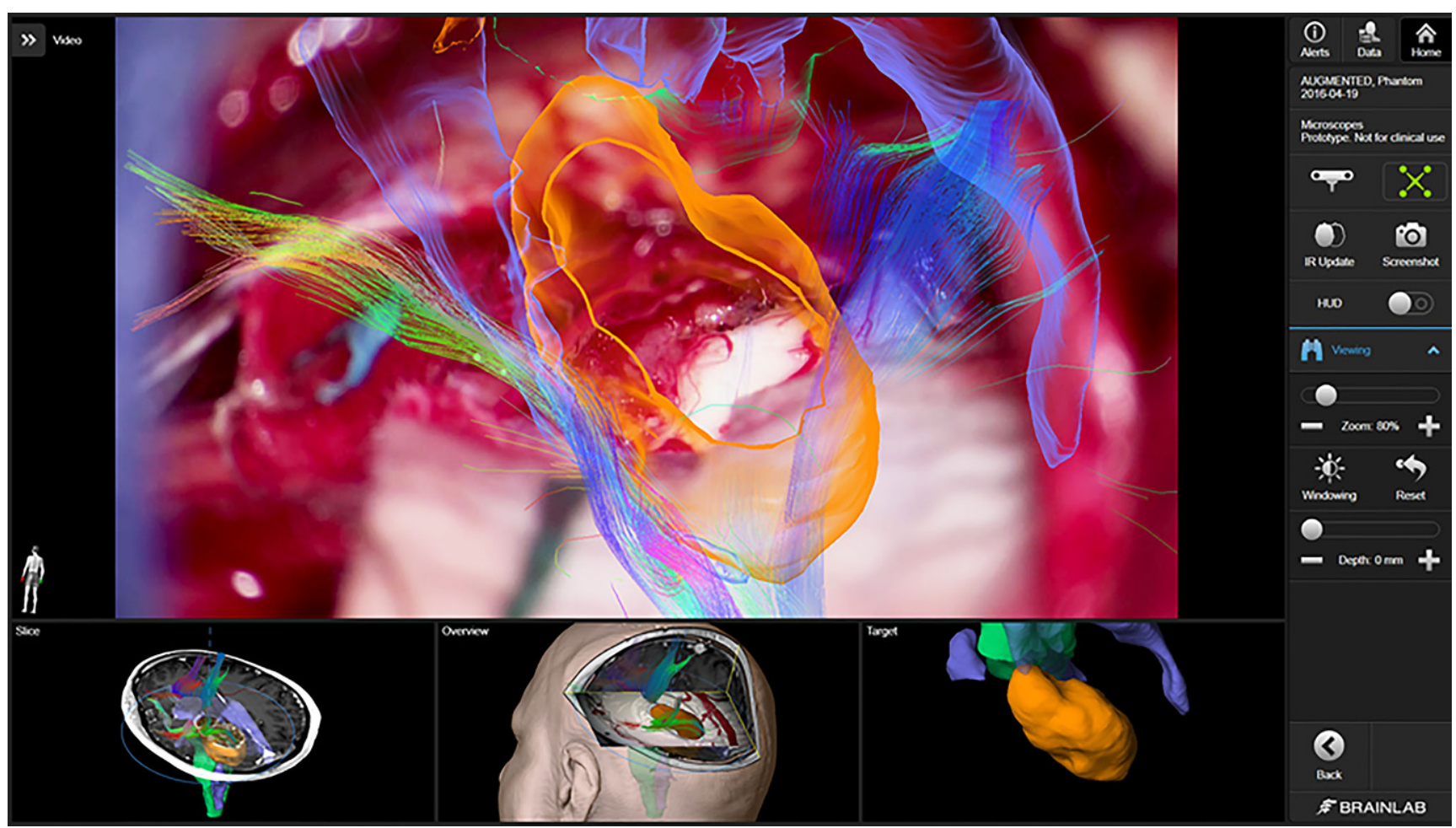

Abb. 1 ॥ Die Software berechnet Modelle dreidimensional mit farblich abgesetzten definierten Bereichen auf der Basis von CT-oder MRT-Bildern. Somit ist die Lage von Gefäßen und Fasern im Gehirn für den Operateur erkennbar. (Mit freundl. Genehmigung $\odot$ Brainlab AG, München)

denen sich Instrumente verschiedener Hersteller integrieren lassen. Möglich ist dies beispielsweise mithilfe einer Kalibriermatrix, mit der sämtliche rigiden Instrumente von Drittherstellern navigierbar sind. Im HNO-Umfeld wird dies v. a. für Endoskope, Debrider, Sauger und Blakesly-Zangen genutzt. In einem digitalisierten OP können Chirurgen zum Beispiel in der funktionellen endoskopischen Nasennebenhöhlenchirurgie (FESS) zu jedem Zeitpunkt eines Eingriffs die Position ihrer Instrumente überprüfen.

Je nach Eingriffsart und Vorlieben sind 2 unterschiedliche Navigationstechnologien auf dem Markt verfügbar - die optische oder die elektromagnetische (EM-)Navigation. Der Einsatz beider Technologien stellt sicher, dass betroffenes Gewebe vollständig reseziert wird und sich Komplikationsrisiken reduzieren lassen. Sowohl die optische als auch die elektromagnetische Navigation bieten hierbei eine hohe Genauigkeit.

Die Technologie der optischen Navigation basiert auf der Sichtbarkeit reflektierender Markerkugeln, die am Patien- ten selbst sowie allen Instrumenten angebracht sind. Von einer Kamera gesendetes Infrarotlicht reflektiert an diesen Kugeln. Die dabei gewonnenen Werte sind die Basis für das Navigationssystem, um die Position der Instrumente in Relation zum Patienten zu bestimmen. Nachteil dieser Technologie kann die unterbrochene Sichtlinie zwischen Instrument und Kamera sein. Dies geschieht beispielsweise durch Op.-Personal im Raum oder durch mit Blut verunreinigte Markerkugeln.

Bei der elektromagnetischen Navigation - hierfür werden kleine Spulen am Patienten sowie an allen Instrumenten angebracht - bedarf es keines Sichtkontakts zwischen Signalgeber und -empfänger. Das System nutzt einen Feldgenerator, der die Bewegung der Spulen im Magnetfeld detektiert. Somit kann das Navigationssystem die Position der Instrumente in Relation zum Patienten bestimmen. Gut geeignet ist die elektromagnetische Navigation für Eingriffe mit Endoskopen und v. a. bei der 4-HandChirurgie. Im Gegensatz zur optischen Navigation gibt es hier keine Sichtlinie, die gestört werden kann. Zudem ist es dem Chirurgen möglich, mithilfe der Remote-Funktionalität des EM-Pointers alle Registrierungsschritte zu absolvieren, ohne den Navigationsbildschirm zu berühren.

Neben der schnellen Oberflächenregistrierung durch einen EM-Pointer sichern moderne Navigationssysteme eine Registrierung anatomischer Landmarken. Außerdem können sie DonutMarker halbautomatisch erkennen, 3-DSchwellenwerte für optimierte Registrierungsergebnisse ermitteln, Patientendaten zur Erkennung des Registrierbereichs dreidimensional anzeigen sowie visuelle und akustische Informationen zum Registrierstatus liefern.

\section{Herausforderungen}

\section{Op.-Kommunikation}

Eine effektive Kommunikation ist sowohl innerhalb als auch außerhalb des OP unerlässlich. Insbesondere im OP ist ein hohes $\mathrm{Maß}$ an Koordination zwingend erforderlich, um risikoangepasste Morbidität zu verringern und Zwischenfälle 


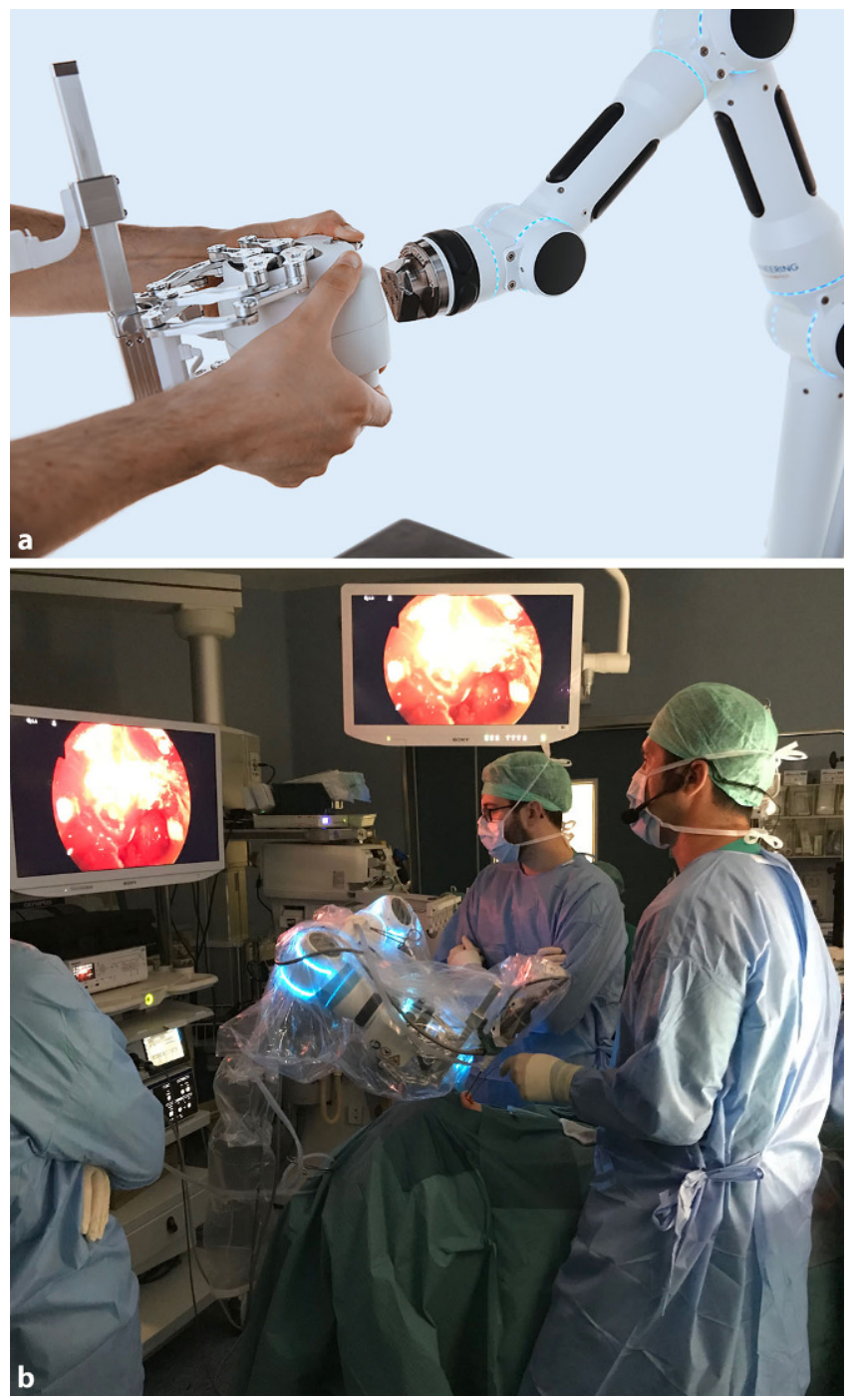

Durch schnelles Streaming über digitale Schnittstellen („serial digital interface", SDI) können zudem Studierende und Weiterbildungsassistenten auch außerhalb des OP Eingriffe in Echtzeit mit voller Hochauflösung, also in Full-HDQualität, mit verfolgen und dies häufiger und bei mehreren Op., ohne dass dabei ein zusätzliches Infektionsrisiko für den Patienten entsteht [4]. Außerdem ermöglicht das Streaming den Chirurgen, die neuesten Op.-Techniken und Ergebnisse bei akademischen Veranstaltungen, Kongressen und Expertentreffen mit Kollegen zu teilen.

\section{Op.-Dokumentation}

Mit einem Touchscreen als einziger zentraler Schnittstelle gelingt es ganz leicht, einen klaren Überblick über alle Patientendaten zu behalten. Digitale Op.-Säle können dem Op.-Team während des gesamten Arbeitsablaufs potenziell viel Zeit und Geld sparen, sowohl innerhalb als auch außerhalb des OP.

Während des Eingriffs kann der Chirurg Videos aufnehmen oder Screenshots von angeschlossenen externen Videoquellen, wie Endoskopen, Mikroskopen oder Op.-Leuchtenkameras, sowie von spezifischen Planungs- und Navigationsapplikationen anfertigen. Auch Informationen aus Op--Tracking-Applikationen, beispielsweise für Op.-Sicherheitschecklisten oder zur Zeiterfassung, werden automatisch in den Patientendatensatz im KIS exportiert und stehen sofort nach dem chirurgischen Eingriff zur Verfügung.

Nach dem chirurgischen Eingriff wird die Dokumentation automatisch oder manuell in das bevorzugte Bildarchiv oder an einen Speicherort, wie PACS, KIS, VNA (Von-Neumann-Architektur), USB-Laufwerk (,universal serial bus"), Quentry-Cloud-Services, übertragen und ist über das KIS unmittelbar auf allen Client-PCs oder angeschlossenen iPads zur Überprüfung oder Patientenberatung verfügbar.

Op.-Aufzeichnungen können zunächst auf einem temporären skalierbaren Storage-Server gespeichert, bis sie mit einem Video Editor bearbeitet werden. So wird sichergestellt, dass nur fundierter Entscheidungen treffen $\mathrm{zu}$ können. Das spart den Experten und Spezialisten außerhalb des OP Zeit. 


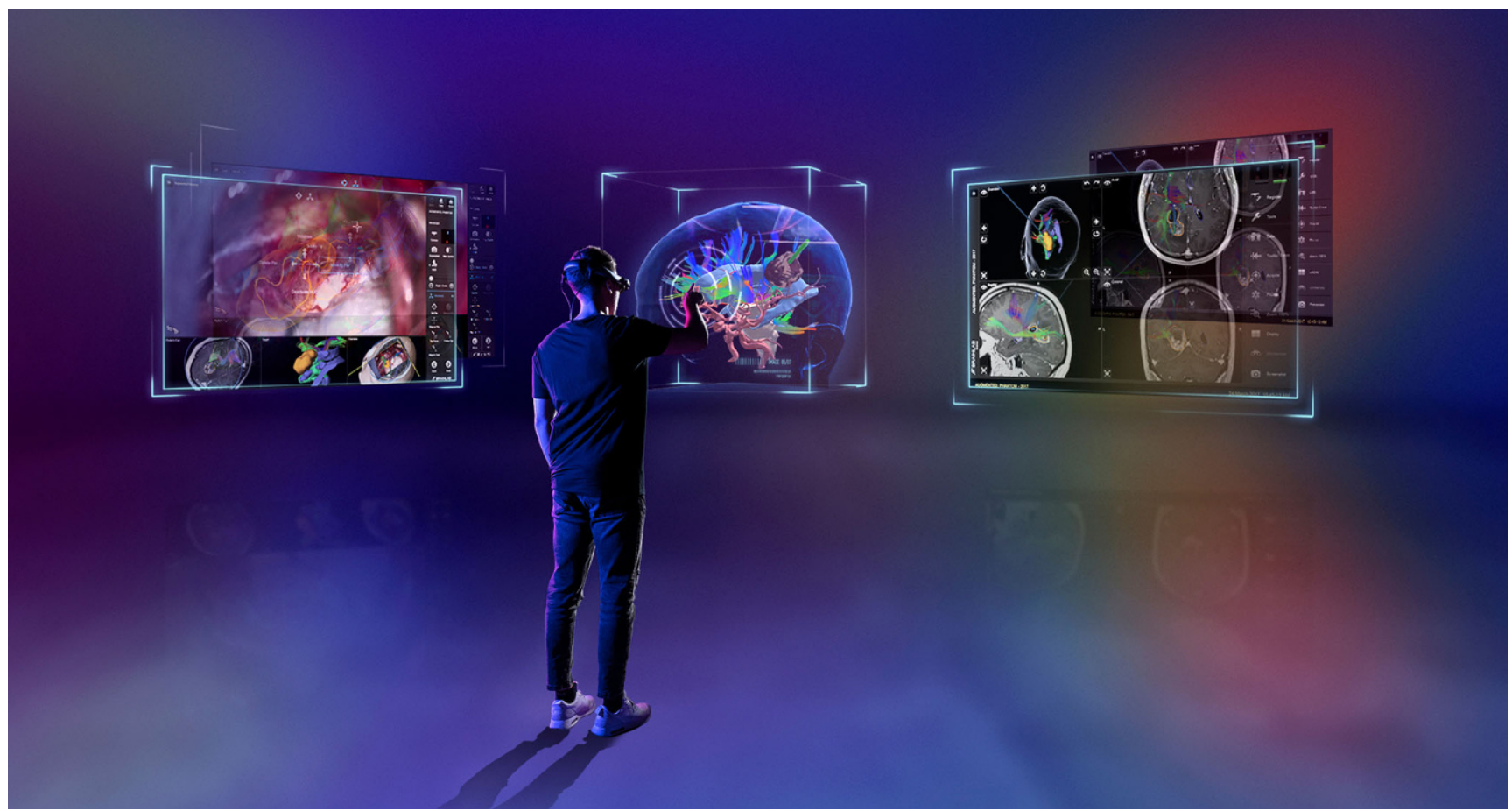

Abb. 3 ॥ Verbindung realer und virtueller Informationen durch Mixed-Reality-Technikund somit Erleichterung für Chirurgen bei der Planung und Durchführung von Operationen. (Mit freundl. Genehmigung $\odot$ Brainlab AG, München)

relevante Daten in den Patientendatensatz im KIS gelangen oder als MP4Format heruntergeladen werden.

Diese fortschrittlichen Technologien zur Unterstützung des Op.-Teams führen jedoch gleichzeitig auch zu einer Zunahme des Datenvolumens und der detaillierten Einzeldaten. Wenn diese Informationsflut nicht ordnungsgemäß verwaltet wird, kann dies zu größeren Verzögerungen vor, während und nach dem chirurgischen Eingriff sowie zu Schwierigkeiten bei der Op.-Dokumentation führen. Aus IT-Perspektive können unkontrollierte Patientendaten Prozesse verlangsamen und wichtigen Speicherplatz im Informationssystem des Krankenhauses belegen.

\section{Assistenzsystem für optimale Sicht}

In einigen Bereichen der Chirurgie profitieren Operateure bereits von robotischen Assistenzsystemen. In der HNOChirurgie ist dies noch nicht der Fall. Das Münchner Unternehmen Medineering möchte das mit einer neu entwickelten modularen Robotik-Plattform ändern. Mithilfe eines mechatronischen Po- sitionierarms, der direkt am Op.-Tisch angebracht und mit verschiedenen applikationsspezifischen Robotern verbunden werden kann, soll ein Portfolio an verschiedenen Robotik-Lösungen für die HNO-Chirurgie entstehen (• Abb. 2). Der von Fa. Medineering entwickelte, direkt am Op.-Tisch angebrachte mechatronische Positionierarm ist seit einem Jahr in mehreren HNO-Kliniken zum Halten und Bewegen des Endoskops im Einsatz. „Mit jedem neuen kleinen Roboter eine neue Robotik-Lösung" das ist das modulare Produktkonzept des Startups. Die erste dieser Lösungen ist bereits am Markt und unterstützt Chirurgen bei endoskopischen HNOOperationen sowie bei der transnasalen Schädelbasischirurgie. Seit Februar 2018 wurde sie rund 100-mal klinisch eingesetzt, u. a. in den Universitätsklinika Essen und Ulm sowie im italienischen Brescia.

Die aus der Kombination von Positionierarm, Roboter und Fußpedal bestehende Lösung entlastet HNO-Chirurgen vom dauerhaften Halten und Bewegen des Endoskops. Dies übernimmt der kleine „Endoscope Robot“. Gesteuert wird das Endoskop aber nach wie vor vom Chirurgen selbst - mithilfe eines Pedals, das direkt am Positionierarm angeschlossen ist. Der Operateur erhält somit einen dritten Arm zum Endoskopieren frei von Tremor und Ermüdung. Das hat sich bisher v. a. bei HNO-Operationen mit knöchernen Strukturen bewährt, wie beispielsweise der Schädelbasis und dem Innenohr. Somit ermöglicht die Lösung erstmals, dass ein einzelner Chirurg beidhändig endoskopisch im Innenohr oder an der Schädelbasis operiert.

\section{Ausblick}

Chirurgen profitieren im Routinebetrieb komplett digitalisierter Op.-Säle derzeit v. a. durch die Möglichkeiten der Informationsintegration und der Datenanreicherung. Mit Letzterer lassen sich die für einen Eingriff benötigten Daten unkompliziert und nutzerorientiert abrufen, während die Informationsintegration Operateure bei der Planung und der Navigation unterstützt. Bei der bildlichen Darstellung anatomischer Strukturen haben Chirurgen derzeit v. a. die Möglichkeit, sich Aufnahmen unterschiedlicher Bildgebungsverfahren fusionieren zu lassen und sich auf einem Monitor anzu- 
schauen oder sich ins Blickfeld eines Op.Mikroskops einblenden zu lassen.

\section{I) Künftig werden in tragbare Datenbrillen eingebaute "head- up displays" eingesetzt}

Doch das ist nur die erste Stufe auf dem Weg in die Welt von Mixed Reality oder Spatial Computing im OP. Künftig werden in tragbare Datenbrillen eingebaute "head-up displays“ (HUD) eingesetzt (• Abb. 3). Sie ermöglichen dem Chirurgen, nicht nur in der realen Welt zu agieren, sondern gleichzeitig auch in der virtuellen. Auf diese Weise können Operateure beispielsweise einzelne Schritte eines komplexen Eingriffs im OP selbst planen, in dem sie die entscheidenden Handgriffe an einem dreidimensionalen Modell ausführen, das nicht nur den individuellen anatomischen Gegebenheiten des Patienten entspricht, sondern das sich genau in der Position des später auf dem Op.-Tisch gelagerten Körpers befindet. Gegenüber der bisher im Vorfeld einer Op. erfolgten Interpretation von zweidimensionalen Bildern auf einem Bildschirm sorgen Lösungen aus dem Bereich der Mixed Reality damit für große Fortschritte. Bei komplexen Eingriffen an kritischen Strukturen kann das virtuelle, den Operateuren mit dem HUD im Raum angezeigte 3-D-Modell die Definition der richtigen Vorgehensweise so erheblich erleichtern.

Der Einsatz der über HUD angezeigten Mixed Reality ermöglicht es, dass mehrere Personen gleichzeitig an dem Objekt arbeiten - und das in der realen Umgebung des OP. Die so gewonnenen Erkenntnisse über die Lage eines Organs, eines Tumors sowie der Blutgefäße bieten ein großes Potenzial. Die Entwickler erwarten, dass diese Systeme die Chirurgen bei ihren Entscheidungen über den weiteren Op.-Verlauf unterstützen können.

\section{Fazit für die Praxis}

- Chirurgen profitieren im Routinebetrieb komplett digitalisierter Op.-Säle derzeit v. a. durch die Möglichkeiten der Informationsintegration und der Datenanreicherung.

- Mit Letzterer lassen sich die für einen Eingriff benötigten Daten unkompliziert und nutzerorientiert abrufen, während die Informationsintegration Operateure bei der Planung und der Navigation unterstützt.

- Bei der bildlichen Darstellung anatomischer Strukturen haben Chirurgen derzeit insbesondere die Möglichkeit, sich Aufnahmen unterschiedlicher Bildgebungsverfahren fusionieren zu lassen und sich auf einem Monitor anzuschauen oder sich ins Blickfeld eines Op.-Mikroskops einblenden zu lassen.

\section{Korrespondenzadresse}

\section{A. Meppelink}

Brainlab AG

Olof-Palme-Straße 9, 81829 München,

Deutschland

auke.meppelink@brainlab.com

\section{Einhaltung ethischer Richtlinien}

Interessenkonflikt. A. Meppelink ist Director Healthcare IT/Digital O.R. Portfolio bei der Fa. Brainlab AG, München.

Für diesen Beitrag wurden von dem Autor keine Studien an Menschen oder Tieren durchgeführt. Für die aufgeführten Studien gelten die jeweils dort angegebenen ethischen Richtlinien.

Open Access. Dieser Artikel wird unter der Creative Commons Namensnennung 4.0 International Lizenz (http://creativecommons.org/licenses/by/4.0/deed. de) veröffentlicht, welche die Nutzung, Vervielfältigung, Bearbeitung, Verbreitung und Wiedergabe in jeglichem Medium und Format erlaubt, sofern Sie den/die ursprünglichen Autor(en) und die Quelle ordnungsgemäßnennen, einen Link zur Creative Commons Lizenz beifügen und angeben, ob Änderungen vorgenommen wurden.

\section{Literatur}

1. Hu YY et al (2012) Deconstructing intraoperative communication failures. J Surg Res 177(1):37-42

2. Lo Presti G et al (2015) Assessment of DICOM viewers capable of loading patient-specific 3D models obtained by different segmentation platforms in the operating. J Digit Imaging 527(5):518

3. Mezrich R, Juluru K, Nagy P (2011) Should postprocessing be performed by the radiologist? J Digit Imaging 24(3):378-381

4. Spagnolo AM (2013) Operating theatre quality and prevention of surgical site infections. J Prev Med Hyg 54(3):131-137
5. Statistisches Bundesamt (2017) Operationen und Prozeduren der vollstationären Patientinnen und Patienten in Krankenhäusern (Wohnort/ Behandlungsort). Gliederungsmerkmale: Jahre, Region, Alter, Geschlecht. OPS 5-224ff 